\title{
Molecular epidemiology and antimicrobial susceptibility of carbapenemase-producing extraintestinal Escherichia coli in a tertiary care hospital of Dhaka, Bangladesh.
}

\author{
Fatima Afroz ${ }^{1}$, Shaheda Anwar', Rokshana Akhtar', Ahmed Abu Saleh ${ }^{1}$ \\ ${ }^{1}$ Department of Microbiology and Immunology, Bangabandhu Sheikh Mujib Medical University, Dhaka, Bangladesh.
}

\begin{abstract}
The emergence of carbapenemase producing Escherichia coli, the first hospital and community-acquired opportunistic pathogen now become a great public health concern. A total of 23 clinical isolates of carbapenemase producing $E$. coli from extraintestinal infections in a tertiary care hospital of Bangladesh were studied. Highest percentage of carbapenemase producing $E$. coli isolates were from urine samples (52.2\%) followed by $21.7 \%$ from each of wound swab and pus and 4.1 $\%$ from blood samples. Among the $E$. coli isolates $69.6 \%$ were from indoor patients and $30.4 \%$ were from outdoor patients. All the isolates (100\%) were positive for $N D M$ of which $13 \%$ were $N D M$ and $O X A-48$ co-producers by conventional PCR. Carbapenemase producing $E$. coli isolates were resistant to most of the antibiotic tested except for nitrofurantoin, colistin, polymyxin B and tigecycline with a sensitivity of $66.7 \%, 82.6 \%, 95.7 \%$ and $100 \%$ respectively.
\end{abstract}

Key wards: Escherichia coli, carbapenemase, NDM, OXA-48, molecular epidemiology, antimicrobial resistance, Bangladesh.

\section{Introduction}

Escherichia coli (E. coli), one of the important nosocomial pathogens of Enterobacteriaceae family is a common etiological factor of urinary tract infections, gastroenteritis, sepsis, meningitis, pneumonia, blood stream infections, intra-abdominal infections and surgical site infections ${ }^{1,2}$. The treatment of infections caused by $E$. coli, especially carbapenemase producing $E$. coli is challenging, because it confers resistance to most $\beta$-lactams including carbapenems and often carries additional antimicrobial resistance genes, making them resistant to most of the antibiotics ${ }^{1,3}$. Recently, carbapenem resistant $E$. coli isolates with limited therapeutic possibilities have been implicated in both hospital and community-acquired infections which became a major health problem all over the world ${ }^{3}$. Among the two types of carbapenemases (serine carbapenemase and metallo- $\beta$-lactamase), New Delhi metallo- $\beta$-lactamase (NDM) and carbapenem-hydrolyzing oxacillinase-48 (OXA-48) are the most common carbapenemases among $E$. coli worldwide ${ }^{4}$. Over the last decade, $N D M$ producers with susceptibility to a few antibiotics including colistin have undergone rapid spread in the South-Asian continent ${ }^{5}$. OXA-48- producing E. coli isolates were also reported in South Asia. The first isolates of $O X A-48$

Correspondence:

Dr. Fatima Afroz

Mobile: 01719395686

E-mail: fatima.mitu@gmail.com producing $E$. coli reported in Japan were isolated from a patient with a medical history in Southeast Asia ${ }^{6}$. In this study, molecular detection of resistant genes (NDM, OXA-48, KPC, IMP) along with antimicrobial susceptibility of carbapenem resistant extraintestinal $E$. coli isolates were carried out.

\section{Materials and Methods}

Bacterial isolates: This cross-sectional study was conducted in the Department of Microbiology and Immunology of Bangabandhu Sheikh Mujib Medical University, Dhaka, Bangladesh from September, 2018 to August, 2019. A total of 23 strains of carbapenem resistant E. coli isolated from different clinical specimens such as urine, blood, wound swab and pus were included in this study. Isolates of $E$. coli were identified by standard biochemical methods. This study was ethically approved by Institutional Review Board (IRB) of Bangabandhu Sheikh Mujib Medical University.

Antimicrobial susceptibility testing: Antimicrobial susceptibility of 23 strains of $E$. coli against 21 different antibiotics were performed on Mueller-Hinton agar (MHA) plates by the modified Kirby-Bauer disc diffusion method using antibiotic discs from BioMaxima, Poland. Results were interpreted according to the criteria of the Clinical and Laboratory Standards Institute (CLSI) $2019^{7}$ guidelines; for polymyxin B and colistin according to CLSI $2007^{8}$ and tigecycline according to EUCAST $2016^{9}$ guidelines. 
Detection of carbapenemase genes by PCR:

The presence of carbapenemase genes were determined by conventional PCR. Bacterial DNA was extracted by boiling method of DNA extraction ${ }^{10}$ and was stored at $-20^{\circ} \mathrm{C}$. All the isolates of E. coli were screened for the presence of $N D M$, $O X A-48, K P C$ and IMP gene separately by using primers described in Table I.

Table I: Primers used for detection of carbapenemase genes.

\begin{tabular}{llllc}
\hline Enzyme & Primer name & Sequence $\left(\mathbf{5}^{\prime}\right.$ to 3') & $\begin{array}{c}\text { Amplicon } \\
\text { size (bp) }\end{array}$ & Reference \\
\hline NDM & NDM forward & ATG GAA TTG CCC AAT ATT ATG CAC & 813 & 11 \\
& NDM reverse & TCA GCG CAG CTT GTC GGC & & \\
OXA-48 & OXA-48 forward & TTGGTGGCATCGATTATCGG & 743 & 12 \\
& OXA-48 reverse & GAGCACTTCTTTTGTGATGGC & & \\
\multirow{2}{*}{$\boldsymbol{P C}$} & KPC forward & ATGTCACTGTATCGCCGTCT & 887 & 13 \\
& KPC reverse & TTTTCAGAGCCTTACTGCCC & & \\
IMP & $I M P$ forward & GAATAG(A/G)(A/G)TGGCTTAA(C/T)TCT & 188 & 14 \\
& $I M P$ reverse & CCAAAC(C/T)ACTA(G/C)GTTATC & & \\
\hline
\end{tabular}

Statistical Analysis: All the data were analyzed using Microsoft Excel 2019. Descriptive analysis of all relevant categorical variables was done by using frequency and percentage.

Results: All E. coli (n=23) isolates were found to be carbapenem (imipenem, meropenem and ertapenem) resistant by the disc diffusion method. Source of carbapenemase producing E. coli isolates were shown in Table II.

Table II: Source of carbapenemase producing extraintestinal E. $\operatorname{coli}(\mathbf{n}=23)$

\begin{tabular}{lcc}
\hline Samples & Number & Percentage (\%) \\
\hline Urine & 12 & 52.2 \\
Wound swab & 5 & 21.7 \\
Pus & 5 & 21.7 \\
Blood & 1 & 4.4 \\
\hline Total & 23 & 100.00 \\
\hline
\end{tabular}

Among the isolates of carbapenemase producing E. coli, $69.6 \%$ $(n=16)$ were isolated from indoor patients and $30.4 \%(n=7)$ were isolated from outdoor patients. Of these 23 patients, $60.9 \%(n=14)$ were male and $39.1 \%(n=9)$ were females. Among tested carbapenemase encoding genes (NDM, OXA-48, $I M P, K P C$ ), only NDM and $O X A-48$ were detected by conventional PCR. Eighty seven percent of carbapenemase producing isolates were positive for only $N D M$ and $13 \%$ were positive for both NDM and $O X A-48$ (Table III). No KPC and IMP genes were detected in any isolates of $E$. coli. Antimicrobial resistance pattern of carbapenemase producing E. coli isolates are shown in Table IV.

Table III: Target genes in carbapenem resistant $E$. coli isolates $(\mathbf{n}=\mathbf{2 3})$

\begin{tabular}{lcc}
\hline Target genes & Numbers of $\boldsymbol{E . c o l i}$ isolates & Percentage (\%) \\
\hline Only $\boldsymbol{N D M}$ & 20 & 87.0 \\
NDM and & 3 & 13.0 \\
$\boldsymbol{O X A}-\mathbf{- 4 8}$ co-producers & & \\
\hline
\end{tabular}

\begin{tabular}{lll}
\hline Total & 23 & 100.0
\end{tabular}

Table IV: Antimicrobial resistance pattern of carbapenemase producing extraintestinal $E$. coli isolates

\begin{tabular}{|c|c|c|}
\hline \multirow[t]{2}{*}{$\begin{array}{l}\text { Antimicrobial } \\
\text { agents }\end{array}$} & \multicolumn{2}{|c|}{$\begin{array}{l}\text { No. of resistant isolates by } \\
\text { carbapenemase encoding genes }\end{array}$} \\
\hline & $\begin{array}{l}\text { Only } N D M \\
(\mathbf{n}=\mathbf{2 0}) \mathbf{n}(\%)\end{array}$ & $\begin{array}{l}N D M \text { and } O X A-48 \\
\text { co-producers } \\
(\mathrm{n}=3) ; \mathrm{n}(\%)\end{array}$ \\
\hline Amoxicillin & $20(100.0)$ & $3(100.0)$ \\
\hline Amikacin & $11(55.0)$ & $3(100.0)$ \\
\hline Aztreonam & $20(100.0)$ & $3(100.0)$ \\
\hline Carbapenems & $20(100.0)$ & $3(100.0)$ \\
\hline Cephalosporins & $20(100.0)$ & $3(100.0)$ \\
\hline Ciprofloxacin & $20(100.0)$ & $3(100.0)$ \\
\hline Cotrimoxazole & $18(90.0)$ & $2(66.7)$ \\
\hline Gentamicin & $15(75.0)$ & $3(100.0)$ \\
\hline Mecillinam & $19(95.0)$ & $3(100.0)$ \\
\hline Nalidixic acid & $20(100.0)$ & $3(100.0)$ \\
\hline Netilmicin & $11(55.0)$ & $3(100.0)$ \\
\hline $\begin{array}{l}\text { Nitrofurantoin } \\
\text { (urinary isolates) }\end{array}$ & $4(36.4), n=11$ & $0(0.0), n=1$ \\
\hline Colistin & $2(10.0)$ & $2(66.0)$ \\
\hline $\begin{array}{l}\text { Piperacillin+ } \\
\text { tazobactum }\end{array}$ & $20(100.0)$ & $3(100.0)$ \\
\hline Polymyxin B & $1(5.0)$ & $\mathbf{0}(\mathbf{0 . 0})$ \\
\hline Tigecycline & $0(\mathbf{0 . 0})$ & $0(\mathbf{0 . 0})$ \\
\hline
\end{tabular}

Note: Carbapenems include meropenem, imipenem, ertapenem and cephalosporins include cefuroxime, ceftriaxone, ceftazidime and cefotaxime.

\section{Discussion}

Carbapenems are the first-choice of treatment for ESBL producing $E$. coli which are resistant to third-generation cephalosporins. 
Widespread use of carbapenems results in the emergence of carbapenem resistant $E$. coli which is attributed to the production of carbapenemases and /or decrease in permeability of the outer membrane ${ }^{1,3}$. In this study $100 \%(\mathrm{n}=23)$ of carbapenem resistant E. coli were found to be positive for NDM of which $13.0 \%$ $(\mathrm{n}=3)$ were co-producers of $N D M$ and $O X A-48$. A study in Bangladesh also reported $N D M$-producing uropathogenic $E$. coli $^{5}$. Khajuria et al (2014) reported $100 \%$ of NDM and 55\% of $N D M$ and $O X A-48$ co-producers among multidrug resistant urinary $E$. coli isolates ${ }^{15}$. The prevalence of $N D M$-producing E. coli in India, China, Pakistan and Nepal were 50.3\%, 21.4\%, $7.4 \%$ and $6.8 \%$ respectively ${ }^{16}$. Easy access to broad-spectrum antibiotics without proper prescriptions, poor sanitation, increased medical tourism for health care and lack of stringent antibiotic are the risk factors for the emergence and spread of $N D M$-producing superbugs in the Indian subcontinent ${ }^{16}$.

Highest percentage of carbapenemase producing extraintestinal E. coli were isolated from urine samples $(52.2 \%)$, followed by wound swab and pus (21.7\%). NDM and OXA-48 carbapenemase producing E. coli isolated from urine, blood, wound swab, cerebrospinal fluids were also reported in other studies ${ }^{15,17}$. All of the isolates included in this study were $100 \%$ resistant to most of the tested antibiotics (amoxicillin, aztreonam, carbapenems, cephalosporins, ciprofloxacin, nalidixic acid, piperacillin+ tazobactum). Currently polymyxins, aminoglycosides and tigecycline are regarded as the main treatment options for the treatment of invasive CRE infections ${ }^{18}$. In their study resistance to the most effective agents, colistin, polymyxin B and aminoglycosides (amikacin, gentamicin and netilmicin) were $17.4 \%$, $4.3 \%$ and $(60.9 \%, 78.3 \%$ and $60.9 \%)$ respectively. The isolates of $N D M$ and $O X A-48$ co-producers were $100 \%$ resistant to amikacin, gentamicin and netilmicin. All the isolates of this study were $100 \%$ sensitive to tigecycline but the status of colistin and aminoglycoside resistance is of particular concern as they are used in empiric treatment protocols to treat invasive CRE infections. Similar findings of resistance to reserve antibiotics were also reported in previous study ${ }^{18}$. Among the urinary isolates of carbapenemase producing E. coli, $66.7 \%$ were sensitive to nitrofurantoin. $96 \%$ and $40 \%$ susceptibility of carbapenemase producing $E$. coli isolates to nitrofurantoin were also reported in other studies ${ }^{19,20}$. These findings are of great importance because $30.4 \%$ isolates of this study were from outdoor patients and nitrofurantoin is frequently prescribed for urinary tract infections in the community, even without microbiological documentation. Reports of E. coli harboring NDM-1 and $O X A-48$ from various parts of the world are a major concern because these genes are located in plasmids which can horizontally transfer between bacteria ${ }^{5}$.

\section{Conclusion}

Being a nosocomial pathogen, E. coli are responsible for both community and hospital-acquired infections, thus raising the fear of dissemination of carbapenemase producing $E$. coli in the community. Emergence of carbapenemase producing strains of $E$. coli with a sensitivity to fewer antibiotics is a very serious concern, especially in a developing country like Bangladesh which frequently experiences antibiotic misuses.

Acknowledgements: This study was supported by Bangabandhu Sheikh Mujib Medical University.

Competing Interests: No conflicts of interest associated with this manuscript.

\section{References}

1. Ojdana D, Sacha P, Olszańska D, Majewski P, Wieczorek P, Jaworowska J, et al. First report of Klebsiella pneumoniaeCarbapenemase-3-producing Escherichia coli ST479 in Poland. BioMed research international. 2015 Aug 3;2015.

2. Solgi H, Giske CG, Badmasti F, Aghamohammad S, Havaei SA, Sabeti S, et al. Emergence of carbapenem resistant Escherichia coli isolates producing blaNDM and blaOXA-48-like carried on IncA/C and IncL/M plasmids at two Iranian university hospitals. Infection, genetics and evolution. 2017 Nov 1;55 :318-23.

3. Zaki M, El-Halaby H, Elmansoury E, Zeid M, Khaled K, Nomir M. Genetic Study of Extended Spectrum Beta-Lactamase and Carbapenemase Producing Escherichia coli Causing Sepsis among Egyptian Children. The Open microbiology journal. 2019 May 31;13(1).

4. Peirano G, Bradford PA, Kazmierczak KM, Badal RE, Hackel M, Hoban DJ, et al. Global incidence of carbapenemaseproducing Escherichia coli ST131. Emerging infectious diseases. 2014 Nov;20(11):1928.

5. Hossain M, Tabassum T, Rahman A, Hossain A, Afroze T, Momen AMI, et al. Genotype-phenotype correlation of $\beta$-lactamase-producing uropathogenic Escherichia coli (UPEC) strains from Bangladesh. Scientific reports. 2020 Sep 3;10(1):1-3.

6. Nishida S, Asahara M, Nemoto K, Ishigaki S, Furukawa T, Sano K, et al. Emergence of Escherichia coli producing OXA-48-like carbapenemase in a patient with percutaneous transhepatic biliary drainage. Infection prevention in practice. 2019 Mar;1(1).

7. Wayne PA. Clinical and laboratory standards institute (CLSI). Performance standards for antimicrobial susceptibility testing. Twenty-nine informational supplement M100-S29. United States, Wayne. 2019.

8. Wayne PA. Clinical and Laboratory Standards Institute (CLSI). Performance standards for antimicrobial susceptibility testing. Seventeenth informational supplement. CLSI Document; M100-S17, United States,2007:32-37. 
9. EUCAST. The European Committee on Antimicrobial Susceptibility Testing. Breakpoint tables for interpretation of MICs and zone diameters. Version 7.1.2016.

10. Dashti AA, Jadaon MM, Abdulsamad AM, Dashti HM. Heat treatment of bacteria: a simple method of DNA extraction for molecular techniques. Kuwait medical journal. 2009 Jun 1;41(2):117-22.

11. Hornsey M, Phee L, Wareham DW. A novel variant, NDM-5, of the New Delhi metallo- $\beta$-lactamase in a multidrugresistant Escherichia coli ST648 isolate recovered from a patient in the United Kingdom. Antimicrobial agents and chemotherapy. 2011 Dec;55(12):5952-4.

12. Poirel L, Héritier C, Tolün V, Nordmann P. Emergence of oxacillinase-mediated resistance to imipenem in Klebsiella pneumoniae. Antimicrobial agents and chemotherapy. 2004 Jan;48(1):15-22.

13. Bratu S, Tolaney P, Karumudi U, Quale J, Mooty M, Nichani S, et al. Carbapenemase-producing Klebsiella pneumoniae in Brooklyn, NY: molecular epidemiology and in vitro activity of polymyxin B and other agents. Journal of antimicrobial chemotherapy. 2005 Jul 1;56(1):128-32.

14. Mendes RE, Kiyota KA, Monteiro J, Castanheira M, Andrade SS, Gales AC, et al. Rapid detection and identification of metallo- $\beta$-lactamase-encoding genes by multiplex real-time PCR assay and melt curve analysis. Journal of clinical microbiology. 2007 Feb;45(2):544-7.

15. Khajuria A, Praharaj AK, Kumar M, Grover N. Emergence of Escherichia coli, co-producing NDM-1 and OXA-48 carbapenemases in urinary isolates at a tertiary care centre at Central India. Journal of clinical and diagnostic research: JCDR. 2014 Jun;8(6):DC01.
16. Dadashi M, Yaslianifard S, Hajikhani B, Kabir K, Owlia P, Goudarzi M, et al. Frequency distribution, genotypes and prevalent sequence types of New Delhi metallo- $\beta$ lactamase-producing Escherichia coli among clinical isolates around the world: a review. Journal of global antimicrobial resistance. 2019 Dec 1;19:284-93.

17. Ranjan A, Shaik S, Mondal A, Nandanwar N, Hussain A, Semmler T, et al. Molecular epidemiology and genome dynamics of New Delhi metallo- $\beta$-lactamase-producing extraintestinal pathogenic Escherichia coli strains from India. Antimicrobial agents and chemotherapy. 2016 Oct 21;60(11):6795-805.

18. Uskudar-Guclu A, Guney M, Sig AK, Kilic S, Baysallar M. "Arising Prevalence of OXA-48 producer and OXA-48 with NDM co-producer Strains" Revista Romana de Medicina de Laborator.2019 July;27(3) :319-326.

19. Gauthier L, Dortet L, Cotellon G, Creton E, Cuzon G, Ponties V, et al. Diversity of carbapenemase-producing Escherichia coli isolates in France in 2012-2013. Antimicrobial agents and chemotherapy. $2018 \mathrm{Jul}$ 27;62(8):e00266-18

20. Livermore DM, Warner M, Mushtaq S, Doumith M, Zhang J, Woodford N. What remains against carbapenem-resistant Enterobacteriaceae? Evaluation of chloramphenicol, ciprofloxacin, colistin, fosfomycin, minocycline, nitrofurantoin, temocillin and tigecycline. International journal of antimicrobial agents. 2011 May 1;37(5):415-9. 